\title{
Estructura de los tributos territoriales en la ciudad de Manizales, su incidencia en los procesos de mitigación del riesgo medioambiental y el desarrollo sostenible*
}

\author{
Recibido: 18 de junio de 2020 - Aprobado: 24 de junio de 2020 \\ https://doi.org/10.22395/seec.v23n54a16 \\ Diana Sofía Serna Giraldo* \\ Luz Dary Echeverry Ruíz ${ }^{* * *}$ \\ Julián Andrés Gómez Sánchez ${ }^{* * *}$ \\ Ciro Alfonso Serna Mendoza ${ }^{* * * *}$
}

\section{RESUMEN}

Este trabajo se propone establecer la relación de la tributación territorial de la ciudad de Manizales con los procesos de mitigación de riesgo medioambiental durante la serie cronológica 2014-2017. Se reflexiona cómo Manizales, durante su desarrollo histórico, se ha venido enfrentando a distintos riesgos a causa de su ubicación geográfica vulnerable desde su planeamiento urbano. Los riesgos propios de la ubicación geográfica de la ciudad presentan importantes dilemas. Por esta razón es importante determinar si se necesita destinar más de los recaudos impositivos o destinar nuevos impuestos para responder a la mitigación de los riegos medioambientales de la ciudad. La principal conclusión es que la asignación directa del presupuesto es una herramienta insuficiente para afrontar los riesgos medioambientales.

\section{PALABRAS CLAVE}

Riesgo medioambiental; impuestos; tributos territoriales.

\section{CLASIFICACIÓN JEL} H23, H71, Q51

\section{CONTENIDO}

Introducción; 1. Impuestos municipales en la ciudad de Manizales; 2. Metodología; 3. Análisis de resultados; 4. Conclusiones; Referencias.

Artículo de investigación derivado del trabajo de grado "Estructura de los tributos territoriales en la ciudad de Manizales, durante el período 2014-2017 y su incidencia en los procesos de mitigación del riesgo medioambiental de la ciudad", de la maestria en Tributación, Universidad de Manizales, Manizales, Colombia.

.. Abogada, Universidad de Manizales, Manizales, Colombia. Magister en Tributación, Universidad de Manizales. Manizales, Colombia. Abogada, Manizales, Colombia. Correo electrónico: dssg15@gmail.com. Orcid: https:// orcid.org/0000-0001-8996-5156

‥ Contadora Pública, Universidad de Manizales, Manizales, Colombia. Magister en Tributación, Universidad de Manizales, Manizales, Colombia. Contadora, Manizales, Colombia.Correo electrónico: luzda.8210@gmail.com

.... Contador Público, Universidad de Manizales, Manizales, Colombia. Magister en Administración, Universidad Nacional de Colombia sede Manizales, Manizales, Colombia. Doctorando de Desarrollo Sostenible, Universidad de Manizales, Manizales, Colombia. Docente investigador, Universidad de Manizales, Colombia. Correo electrónico: jgomezs@umanizales.edu.co. Orcid: https://orcid.org/0000-0002-0328-8949

....*Economista, Universidad Pedagógica y Tecnológica De Colombia, Tunja, Colombia. Especialista en Población y Desarrollo Sostenible, Universidad de Chile, Santiago de Chile, Chile. Magíster en Desarrollo Social y Desarrollo Educativo, Universidad Pedagógica Nacional, Tunja, Colombia. Doctor en Ciencias Pedagógicas, Universidad de la Habana, La Habana, Cuba. Postdoctorado en Filosofía de la Ciencia y la Sustentabilidad, Universidad Autónoma de Chapingo, Texcoco, México. Docente investigador. Universidad de Manizales, Manizales, Colombia. Director el Doctorado en Desarrollo Sostenible, Universidad de Manizales, Manizales, Colombia. Correo electrónico: redesomciro@hotmail.com. Orcid: https://orcid.org/0000-0003-3192-9771 


\title{
Structure of the Territorial Taxes in the City of Manizales and its Incidence in the Processes of Mitigation of Environmental Risk and Sustainable Development
}

\begin{abstract}
This work's purpose is to establish the relationship between the territorial taxing of the city of Manizales with the processes of environmental risk mitigation during the chronological series of 2014 to 2017. This article reflects on how Manizales, during its historical development, has been facing different risks because of its vulnerable geographical location since its urban planning. The inherent risks of its geographical location are a source of important dilemmas. For this reason, it is important to assign more tax resources and designate new taxes towards the mitigation of the environmental risks of the city. The main conclusion is that this direct assignment of the budget is an insufficient tool for facing the environmental risks.
\end{abstract}

\section{KEYWORDS}

Environmental risk; taxes; territorial taxes.

\section{JEL CLASSIFICATION}

H23, H71, Q51

\section{CONTENT}

Introduction; 1. Municipalities taxes in the city of Manizales; 2. Methodology; 3. Results analysis;

4. Conclusions; Bibliography.

\section{Estrutura dos impostos territoriais na cidade de Manizales, sua incidência nos processos de redução do risco meio ambiental e o desenvolvimento sustentável}

\section{RESUMO}

Este trabalho propõe estabelecer a relação da tributação territorial da cidade de Manizales, Colômbia, com os processos de redução do risco meio ambiental durante o período de 2014 a 2017. Reflete-se como Manizales, durante seu desenvolvimento histórico, enfrentou diferentes riscos por causa de sua localização geográfica vulnerável a partir de seu planejamento urbano. Os riscos próprios da localização geográfica da cidade apresentam importantes dilemas. Por isso, é importante determinar se é necessário destinar mais das arrecadações obrigatórias ou destinar novos impostos para atender a mitigação dos riscos meio ambientais da cidade. Conclui-se, principalmente, que a designação direta do orçamento é uma ferramenta insuficiente para enfrentar os riscos meio ambientais.

\section{PALAVRAS CHAVE}

Risco meio ambiental; impostos; tributos territoriais.

\section{JEL RATING}

H23, H71, Q51

\section{CONTEÚDO}

Introdução; 1. Impostos municipais na cidade de Manizales; 2. Metodologia; 3. Análise de resultados; 4. Conclusões; Bibliografia. 


\section{INTRODUCCIÓN}

Desde finales del siglo XVIII e inicios del siglo XIX data el origen de la ciudad de Manizales. Este suceso estuvo articulado al proceso de expansión poblacional conocido como "colonización antioqueña". Recién comenzado el siglo XX, acontecimientos como la separación de Panamá y la guerra de los Mil Días llevaron a que el presidente de la época, Rafael Reyes, realizara una serie de reformas de tipo administrativo que propendían por la reestructuración político-administrativa de Colombia. La Ley 11 de 1905 fue una de estas. Su propósito fue contribuir a la segregación de las provincias del sur de Antioquia, de Robledo y Marmato. De esta manera, se crea un nuevo departamento con el nombre de Caldas cuya capital sería Manizales (Alcaldía de Manizales, 2020). Seis décadas después, el viejo Caldas se fragmentó, dando origen a tres departamentos distintos: Risaralda, Quindío y el departamento de Caldas, este último con Manizales como su capital. El trazo de la ciudad de Manizales se hizo de acuerdo con el modelo de colonización española, el cual estaba conformado por una plaza central y, alrededor de esta, se establecían actividades administrativas, religiosas y comerciales (Jiménez, 2013).

De acuerdo con la Alcaldía de Manizales (2020), este municipio ha pasado, desde sus orígenes, por diversos fenómenos de deterioro en el ámbito medioambiental que han afectado el buen vivir de sus habitantes. Este deterioro se ha materializado en desastres que han destruido buena parte del patrimonio arquitectónico de la ciudad, como fue el caso de los incendios de los años 1922, 1925 y 1926, los cuales dejaron en ruinas a su antigua catedral. La reconstrucción de la ciudad se dio para el decenio comprendido entre 1930 y 1940, momento en el que, se construyó la Catedral Basílica de Nuestra Señora del Rosario de Manizales, la que a la fecha es uno de los principales iconos de la ciudad. De igual forma debe advertirse que la ciudad de Manizales, debido a su ubicación, se ha expuesto a una serie de eventos sísmicos que se resumen en: el sismo del 30 de julio de 1962, cuya replica hizo colapsar una de las torres de la catedral; el sismo del 23 de noviembre de 1979, cuyo epicentro se ubicó en el norte del Valle del Cauca; y el sismo del 25 de enero de 1999, cuyo epicentro fue el eje cafetero, en especial la ciudad de Armenia y que, por su cercanía y poca profundidad, dejó daños considerables en la ciudad de Manizales. De igual forma, la erupción del volcán Nevado del Ruíz, si bien no dejó daños graves en Manizales, afectó a sus habitantes al dejar la ciudad cubierta de cenizas debido a que este se encuentra en la zona de influencia de la ciudad (Corporación Autónoma Regional de Caldas, 2013).

Los barrios de la ciudad de Manizales también han sufrido los impactos medioambientales. Por ejemplo, en diciembre de 1993, el barrio La Carolita fue 
afectado por un desprendimiento de tierra que destruyó una veintena de casas, lo que originó la muerte de cinco personas y el cierre de las vías aledañas afectadas por el desastre natural (La Patria, 2012). Del mismo modo, en diciembre del año 2003 hubo un desprendimiento de tierra en el barrio La Sultana que terminó con la vida de dieciséis personas (El Tiempo, 1993). Asimismo, en noviembre de 2011, las fuertes lluvias asociadas al fenómeno de La Niña ocasionaron un desprendimiento de tierra en el barrio Cervantes. Se estima que murieron unas cincuenta personas (La Patria, 2019). De forma más reciente, en abril de 2017, el barrio Aranjuez sufrió una avalancha, también originada por la temporada invernal, en la que se afectaron unas ochenta viviendas y en la que se estima que murieron diecisiete personas (Semana, 2017).

Dentro del plan de desarrollo 2016 a 2019, el alcalde José Octavio Cardona León hace una comparación histórica de todos los recursos que recibe el municipio y cómo ha sido su destinación en los últimos años (Alcaldía de Manizales, 2016, p. 356). Además de un análisis del comportamiento del recaudo, dicha comparación sirvió de base para proyectar los ingresos financieros futuros percibidos por el municipio para poder atender todas las necesidades y contingencias. Este plan de desarrollo plantea la valoración del patrimonio ambiental. Con ello, ayuda a generar conciencia ciudadana con el cuidado que requiere el municipio, tanto en el sector urbano como rural, con el objetivo de que cada forma de vida sea protegida. Todo el recorrido anterior y las reflexiones que se han planteado hacen oportuna la investigación, pues resulta que es necesario pensar y repensar cómo, en el futuro inmediato, se puedan destinar recursos frescos desde el ámbito de los recaudos fiscales municipales tendientes a la mitigación de los riesgos medioambientales de la ciudad (Maya y Rosero, 2015; Murillo, 2016).

Por otro lado, Acquatella y Barcena (2005) señalan que:

en América Latina el creciente reto de diseñar instrumentos económicos que deben permitir hacer una gestión ambiental efectiva desde todo punto de vista, para lograr de esta forma el cumplimiento de las metas trazadas por los países latinoamericanos, en donde se ha impuesto la necesidad de mejorar la calidad ambiental en un menor costo posible, respondiendo adecuadamente a los procesos de deterioro de la calidad del ambiente de la región, y es por ello que las autoridades ambientales de la región se ven limitadas en fortalecer su capacidad presupuestal y deben explorar funciones de autofinanciamiento para así avanzar en la gestión ambiental. (p. 26)

Así las cosas, se plantea la necesidad de tener los recursos financieros para poder avanzar en la mitigación de los riesgos ambientales debido a que su atención demanda inversiones importantes. Asimismo, es importante pensar en los mecanismos de prevención (Rodríguez, 2008). En este sentido, se hace pertinente generar 
la siguiente pregunta: ¿Cuál es la relación de la tributación territorial de Manizales y los procesos de mitigación del riesgo medio ambiental en el periodo 2014-2017? Esta pregunta orienta el presente trabajo.

\section{IMPUESTOS MUNICIPALES EN LA CIUDAD DE MANIZALES}

La información de esta sección se soporta en el Acuerdo 704 del 29 de diciembre de 2008, emanada del Consejo de Manizales (2008), el cual presenta las disposiciones que rigen para los tributos de la ciudad de Manizales.

- Impuesto predial unificado: este gravamen se aplica a los bienes inmuebles ubicados en la ciudad de Manizales. Este impuesto se causa por la existencia del predio y su pago se hace el primero de enero de cada año, por lo que su recaudo es anual. La base gravable para liquidar este impuesto se fija de acuerdo con el avaluó catastral, el cual es fijado por las autoridades catastrales o por medio de un autoavalúo declarado por el propietario. Par el cobro de este impuesto, las tarifas se establecen de acuerdo con los estratos para el sector residencial. Estas están entre el 5,0 y el 8,5 por mil: para el sector no residencial la tarifa oscila entre el 9,5 y 10,5 por mil; para los predios que tengan avalúos oscila entre 392,5 SMMLV hasta 625 SMMLV sin importar el tipo de estrato que tengan. También existen unas tarifas especiales para los predios rurales de tipo recreacional del 9,0 por mil. Por último, para los predios de tipo no recreacional las tarifas están entre el 4,0 y el 6,5 por mil con avalúos mayores a los 375 SMMLV.

- Impuesto de industria y comercio: se establece a todas las actividades directas o indirectas que personas naturales o jurídicas sin o con establecimiento comercial lleven a cabo en la ciudad Manizales. Este impuesto grava, también, todo tipo de ingresos provenientes de honorarios, donaciones, arrendamientos, dividendos y, en general, sobre cualquier rendimiento financiero, teniendo en cuenta la actividad económica declarada por el contribuyente (Acuerdo 704, 2008).

- Impuesto complementario de avisos y tableros: los contribuyentes que tengan avisos o anuncios de su negocio en el espacio público deben liquidar y pagar este impuesto. La tarifa establecida es del $15 \%$ con respecto al impuesto de industria y comercio.

- Sobretasa bomberil: el destino de este es el cuerpo de bomberos de Manizales. Este es pagado solo una vez al año y representa el 50 \% del valor del impuesto de avisos y tableros. 
- Retención a título de industria y comercio: toda entidad del orden nacional, departamental o municipal, así como los grandes contribuyentes, las empresas comerciales del Estado y las sociedades de economía mixta, cuyo domicilio sea el municipio de Manizales, deben efectuar retención del impuesto de industria y comercio, en especial, aquellos abonos que superen un (1) SMMLV y que representen "ingresos por actividades industriales, comerciales, de servicio o financieros sometidos al impuestos de Industria y Comercio en el municipio de Manizales" (Acuerdo 704, 2008).

- Impuesto de circulación y tránsito o rodamiento: los automóviles que se encuentren matriculados en la ciudad de Manizales, tanto particulares como de servicio público, tendrán que pagar una tarifa entre 1,5 y 8 \% según su clasificación.

- Impuesto de delineación urbana: este impuesto se causa cuando se solicita al municipio expedir una licencia de construcción de edificaciones nuevas o en remodelación conforme a las disposiciones legales vigentes. Este impuesto tiene una base gravable del 1 \% del presupuesto requerido para la construcción de la obra.

- Impuesto de espectáculos públicos: este impuesto está comprendido por el cobro del ingreso a un espectáculo que se presente en escenarios públicos u otros sitios donde se congregue público dentro del municipio de Manizales. La tarifa para este impuesto es del 10 \% sobre los ingresos en términos brutos, los cuales están asociados al valor de la boleta.

- Rifas: este impuesto comprende la modalidad de juegos de suerte y azar, el cual debe cumplir con ciertos requisitos como la fecha y el monto del premio. Asimismo, los boletos deben ir en numeración continua y a un precio fijo, y deben ser comercializadas por un operador autorizado. La tarifa del impuesto de rifas es del $14 \%$ de los ingresos por el total de las boletas emitidas.

- Publicación en la gaceta municipal: este impuesto se genera cuando una entidad estatal publique todo tipo de contrato estatal, así como la de aprobación a reforma de estatutos, el reconocimiento o cancelación de personería jurídica y la inscripción de entidades ambientalistas que carezcan de ánimo de lucro, suscrito con el municipio de Manizales. La tarifa para la publicación en la gaceta municipal se liquidará a razón de 0,5 SMDLV por cada fracción de millón de pesos.

- Registro y certificado de propiedad horizontal: la tarifa es equivalente al $20 \%$ del SMDLV. Este impuesto se cobra con el ánimo de reconocer la naturaleza 
jurídica de las entidades ambientalistas sin fines lucrativos y otros registros que el alcalde debe definir por ley. Para la expedición de representación legal y de certificados de existencia expedidos en función de las propiedades horizontales, la tarifa estipulada es de un SMDLV.

- Degüello de ganado menor: es el impuesto cobrado por cabeza de ganado sacrificado en la ciudad de Manizales. Este impuesto equivale al $10 \%$ de un SMDLV.

- Placas, pases y otros de tránsito: todo propietario de vehículo cuya matrícula provenga de la Secretaria de Tránsito del municipio de Manizales debe pagar este impuesto. Estas tarifas oscilan entre 2,5 SMDLV y cien SMDLV.

- Impuesto de publicidad exterior visual: la Secretaría de Hacienda liquida este impuesto que equivale a cinco SMMLV. Este impuesto lo debe pagar todo aquel que instale vallas en la jurisdicción del municipio.

- Coso municipal: impuesto pagado por semoviente en vía pública o en predios de terceros. La tarifa por semoviente en vía pública es de 5,8 \% del SMMLV y por cada día en que este permanezca en el coso municipal su tarifa será el $4 \%$ del SMMLV.

\section{METODOLOGÍA}

La metodología de este trabajo tiene dos momentos. En el primero de ellos se hace una revisión de fuentes secundarias que den cuenta de la relación entre la tributación territorial de Manizales y los procesos de mitigación del riesgo medio ambiental en el periodo 2014-2017. Para ello, se toman como base los planes de desarrollo 2012-2015 y 2016-2019 y sus presupuestos (Alcaldía de Manizales, 2010, 2016) y la estructura tributaria del municipio de Manizales (Acuerdo 704, 2008) para el periodo de estudio.

En un segundo momento se realizaron diez entrevistas semiestructuradas a funcionarios y actores del municipio de Manizales con el fin de conocer la percepción de inversión en el medio ambiente a partir de la estructura tributaria municipal. Las entrevistas se hicieron al exsecretario de hacienda del municipio, el secretario de hacienda, el secretario del medio ambiente y a seis investigadores en el área del objeto de estudio. La tabla 1 muestra las preguntas realizadas. 


\section{Tabla 1. Entrevistas a actores relevantes municipio de Manizales}

1. ¿Cree usted que existe alguna relación entre los impuestos que se reciben en la ciudad de Manizales y la atención a los riesgos medioambientales? Sí o no. ¿Por qué?

2. ¿Cómo considera se ha venido dando en Manizales la relación entre tributación de Manizales y los procesos de mitigación del riesgo en Manizales en el periodo 2014-2018?

3. Si disminuye el recaudo de los impuestos territoriales en la cuidad de Manizales, se afectará de manera significativa el tratamiento a la mitigación de los riegos medioambientales en la ciudad de Manizales. ¿Considera esto posible para una ciudad como Manizales? Sí o No. Explique.

4. Frente a los últimos acontecimientos que se han tenido en la ciudad de Manizales en materia de desastres medioambientales, ¿considera pertinente que se haga más inversión y la mitigación de los riesgos por la vía impositiva? Sí o no. ¿Por qué?

5. ¿Cree que se deberían crear nuevos impuestos en Manizales destinados solo a la mitigación y prevención de riegos? Sí o no. ¿Por qué?

6. ¿Conoce usted cuáles son los desastres y riesgos ambientales de Manizales, dados entre el 2014 y el 2017?

7. ¿Qué propuesta haría usted para que se pudiera establecer una adecuada relación entre impuestos territoriales en Manizales y la atención a los riesgos medio ambientales?

Fuente: elaboración propia.

Las preguntas de las entrevistas fueron validadas mediante la metodología de juicio de expertos.

\section{ANÁLISIS DE RESULTADOS}

Por el uso y el paso del tiempo los recursos tienden a agotarse. Al respecto, el Ministerio del Medio Ambiente y Desarrollo Sostenible, mediante la Ley 99 (1993), plantea que, al representar parte del patrimonio del país, estos deben ser protegidos y aprovechados de forma sostenible teniendo en cuenta el tiempo y el cuidado del recurso. En este sentido, la forma de mitigar su deterioro implica la aplicación de tasas retributivas y compensatorias. Para el caso de la cuidad de Manizales se determinó que el plan de desarrollo 2012-2015 implementó el 0,5 por mil adicional a la sobretasa ambiental recaudada dentro del impuesto predial con destinación a Corpocaldas, con el fin de implementar acciones en materia de mitigación y prevención del riesgo causado por los cambios climáticos en el municipio. 
Ahora bien, de acuerdo con los entrevistados, la relación entre los impuestos territoriales y la mitigación del riesgo medioambiental en Manizales para el período 2014-2017, se puede considerar como una relación inversamente proporcional (Beltrán y Ávila, 2013). Este hecho se puede explicar a partir del eje de las abscisas, el cual se consideró I (impuesto) y el eje de las ordenadas RA (riesgo ambiental). El gráfico 1 muestra una inferencia que se puede deducir de la información proporcionada por los actores.

Gráfico 1. Relación entre los impuestos territoriales y la mitigación de riesgos medioambientales

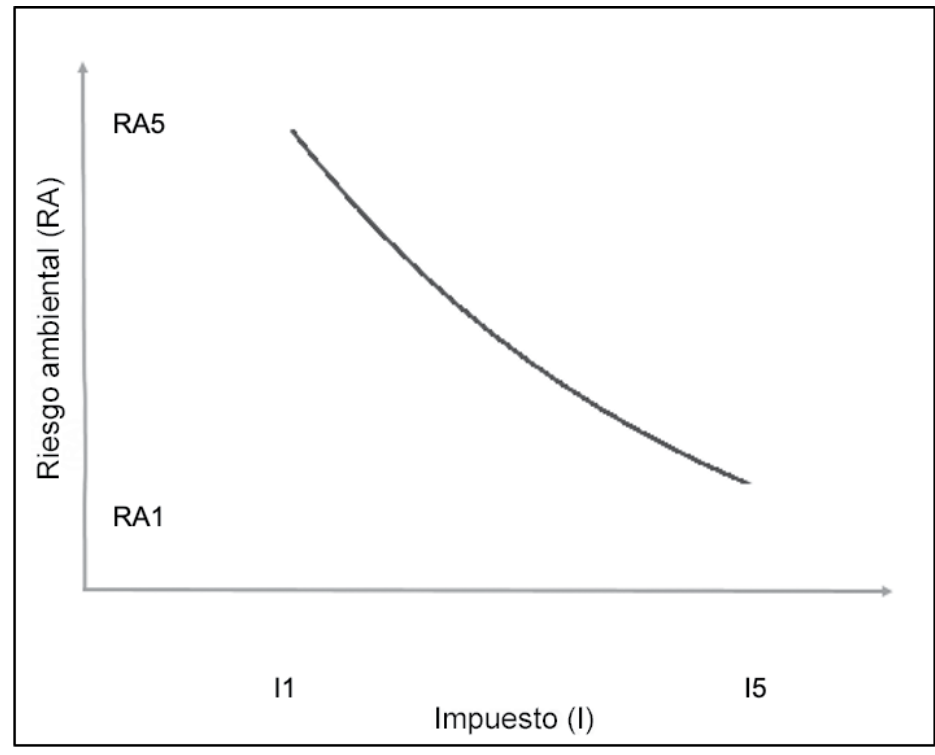

Fuente: elaboración propia.

Este aspecto tiene estrecha relación con lo que se les preguntó a los entrevistados, pues creen que existe alguna relación entre los impuestos que se reciben en la ciudad de Manizales y la atención a los impuestos medioambientales. Se encontró que el $100 \%$ de los entrevistados coinciden en que esta relación existe, pero se infiere de lo respondido que se mantiene una relación inversamente proporcional, tal como se explicó en el gráfico 1.

No obstante, y con base en los encuestados, muchas de las situaciones de desastres en Manizales todavía no están resueltas a cabalidad, pues faltan recursos financieros para atender tales emergencias. En la tragedia de Aranjuez, por ejemplo, ocurrida el 18 de abril de 2017, se presentaron muchas dificultades para tratar de solventar la tragedia. Los recursos se demoraron en llegar y la Alcaldía manifestó 
dificultad para tener recursos inmediatos para atender la emergencia. Esta situación deja ver que los recaudos de la municipalidad son insuficientes para atender situaciones de riesgo en una ciudad que, efectivamente por su ubicación geográfica, está en permanente riesgo.

Por ello, la relación de riesgos ambientales e impuestos territoriales implica abordar la problemática que existe en esta relación y reconocer la diversidad de condiciones territoriales, poblacionales, económicas a nivel de ecosistemas y regionales. Esto lleva a pensar que las estructuras impositivas departamentales deben tratar de contemplar una gestión ambiental encaminada a tener en cuenta aspectos como la deforestación, pérdida de suelos, desertificación, recursos hidrológicos; contaminación que la industria genera en fuentes hídricas, agrícolas y domiciliarias; la inadecuada disposición de residuos sólidos; los desastres naturales y la vulnerabilidad de la población.

Por otro lado, se preguntó a los entrevistados si conocían cuáles eran los riesgos y desastres ambientales ocurridos en Manizales durante el período 2014 y 2017, a lo cual el 100 \% respondió que sí. Ahora bien, se preguntó si deberían colocarse nuevos impuestos en Manizales destinados solo a la mitigación de riesgo. El 90 \% responde afirmativamente (sí), mientras que el $10 \%$ contesta negativamente (no). El argumento que plantean las personas que se han inclinado por la respuesta negativa es que la ciudad tiene otros asuntos que atender, independientemente de los riesgos, las catástrofes y las vulnerabilidades. Así pues, estas personas argumentan que, si en la ciudad se hacen las suficientes prevenciones, no tiene por qué destinarse tanto recurso a la mitigación. Asimismo, se les hizo una contrapregunta acerca de si la prevención implicaba inversión, ante lo que responden que no. Con ello, se infiere que los entrevistados tienen desconocimiento respecto a lo que tiene que ver con la prevención, toda vez que prevenir y mitigar implica también destinar recursos financieros para cumplir con tal fin. Con respecto a lo anterior se entiende lo que plantean Natenzon y Rios (2015) con respecto a los aportes desde la geografía:

...en la literatura de los estudios sobre catástrofes desde hace tiempo se reconoce que el riesgo resulta de la combinación de dos variables o dimensiones: la amenaza y la vulnerabilidad. La primera, también denominada peligro, estaría colocada en el "origen" del problema. Es el proceso o factor disparador del escenario de riesgo. En consecuencia, se supone que estudiando estos fenómenos o procesos desencadenantes que están en su origen estaríamos en condiciones de intervenir y acotar el efecto negativo de esas amenazas o peligros. (p. 227) 


\section{CONCLUSIONES}

A pesar de existir determinados tipos de impuestos, se encontró que estos son insuficientes para asumir los retos de una ciudad como Manizales, la cual debe atender sus situaciones de riesgos ambientales con los que se enfrenta. Esto se logra colegir del proceso de revisión de fuentes secundarias y primarias. Estas últimas se soportan en las entrevistas semiestructuradas realizadas a actores relevantes en la temática tratada en el municipio de Manizales.

El estatuto tributario de Manizales y el estatuto de rentas del departamento tienen una variedad de impuestos que sirven como fuente de ingreso para alcanzar los objetivos de cada rubro del presupuesto municipal. No obstante, se evidenció que solo del impuesto predial se destina un pequeño porcentaje para la mitigación del riesgo medioambiental y atención de desastres del municipio, el cual no alcanza a cubrir todos los perjuicios ocasionando por las catástrofes naturales.

De acuerdo con las entrevistas, se puede decir que la relación entre los impuestos territoriales y la mitigación del riesgo medioambiental en Manizales, para el período 2014-2017, se considera como una relación inversamente proporcional. Esto indica que, a menores impuestos, los riesgos se hacen más altos, es decir, se necesitan más recursos para mitigar los riesgos.

Muchas de las situaciones de desastres en Manizales aún no están resueltas a cabalidad y faltan recursos financieros para atender tales emergencias. Esto explica, de alguna manera, que los recaudos que tiene la municipalidad son insuficientes para atender situaciones de riesgo en una ciudad que, efectivamente, está en permanente riesgo por su ubicación geográfica.

En este sentido, se concluye que se necesitan más recursos financieros, en especial en el campo de los tributos recaudados por el municipio, para generar un plan a corto, mediano y largo plazo con respecto a la mitigación de los riesgos ambientales. Esto se debe a que, como se identificó, la cuidad de Manizales es propensa a sufrir catástrofes, las cuales demanda atenderlas. Este aspecto deja ver que las inversiones deberán ser importantes, así como los mecanismos para lograr prevenir y no llegar hasta la mitigación.

\section{REFERENCIAS}

Acquatella, Jean y Barcena, Alicia (2005). Política Fiscal y medio ambiente: bases para una agenda común. Santiago de Chile: Cepal, 264p.

Alcaldía de Manizales (2010). Plan de desarrollo 2012-2015. Manizales: Alcaldía de Manizales, 387p. 
Alcaldía de Manizales. (2016). Plan de desarrollo 2016-2019. Manizales, Colombia: Alcaldía de Manizales, 426p. https://manizales.gov.co/RecursosAlcaldia/201605021636516132.pdf

Alcaldía de Manizales. (2020). Historia de Manizales. Manizales, Colombia: Alcaldía de Manizales. https://manizales.gov.co/historia/

Beltrán, John Jairo y Ávila, Jesús (2013). Efectos de la tributación ambiental en la gestión del medio ambiente. Medellín: Universidad de Medellín, 123p.

Concejo de Manizales. (2008, 29 de diciembre). Estatuto Tributario de Manizales, Acuerdo 704. Por el cual se codifican las disposiciones que rigen los tributos municipales, y se dictan otras disposiciones. Manizales, Colombia, 17p. http://manizales.gov.co/RecursosAlcaldia/201506041605046114.pdf

Congreso de la República de Colombia (1993). Ley 99 de 1993. Bogotá, Colombia.

Corporación Autónoma Regional de Caldas (2013). Diagnóstico Ambiental De Caldas 2013-2015. Caldas: Corporación Autónoma Regional de Caldas, 141p. http://www.corpocaldas.gov.co/ publicaciones/1257/Diagnostico_del_Plan_de_Accion_2013-2015.pdf

El Tiempo (1993). Derrumbe arrasó 10 casas en Manizales. https://www.eltiempo.com/archivo/ documento/MAM-278325

Jiménez, Oscar Andrés (2013). Subasta del suelo y estructura urbana: un análisis para el área urbana de Manizales. Manizales: Universidad de Manizales, Facultad de Ciencias Contables, económicas y administrativas, 85p.

La Patria (2012). 19 años para hallar culpables de tragedia en el barrio La Carolita. https:// www.lapatria.com/manizales/19-anos-para-hallar-culpables-de-tragedia-en-el-barrio-la-carolita-22409.

La Patria (2019). Tragedia del barrio Cervantes sigue sin responsables. https://www.lapatria.com/ sucesos/tragedia-del-barrio-cervantes-sigue-sin-responsables-445980

Maya, Luís y Rosero, Paola (2015). Los tributos verdes en América Latina: un comparativo. Quito: Congreso Nacional de Profesores de Contaduría Pública, 52p.

Murillo, Luís Gilberto (2016). Reforma tributaria ambiental para un futuro de sostenibilidad. Portafolio. https://www.portafolio.co/opinion/otros-columnistas-1/reforma-tributaria-y-medio-ambiente-columna-minambiente-22-de-diciembre-502425

Natenzon, Claudia y Rios, Diego (2015). Riesgos, catástrofes y vulnerabilidades. Aportes desde la geografía y otras ciencias sociales para casos argentinos. Buenos Aires: Editorial Imago Mundi, 228p.

Rodríguez, Aída (2008). Fundamentos para el uso de instrumentos fiscales en la política ambiental: Una aproximación al caso colombiano. Bogotá: Dian, 101p. https://www.dian. gov.co/dian/cifras/Cuadernos\%20de\%20Trabajo/Fundamentos\%20para\%20el\%20uso\%20 de\%20instrumentos\%20fiscales\%20en\%20la\%20pol\%C3\%ADtica\%20ambiental_Una\%20 aproximaci\%C3\%B3n\%20al\%20caso\%20colombiano..pdf

Semana (2017). Así vivió la tragedia el barrio de Manizales más afectado por deslizamientos. Revista Semana. https://www.semana.com/nacion/articulo/deslizamientos-en-barrio-aranjuez-de-manizales-tres-muertos-un-policia/522718 